\title{
Editorial \\ Special Issue on Light Communications: Latest Advances and Prospects
}

\author{
Anthony C. Boucouvalas ${ }^{1}$, Kostas Yiannopoulos ${ }^{1, *}{ }^{\mathbb{C}}$, Asghar Gholami ${ }^{2}$, Volker Jungnickel ${ }^{3}$ \\ and Zabih Ghassemlooy ${ }^{4}$ (i)
}

1 Communication Networks and Applications Laboratory, Department of Informatics and Telecommunications, Faculty of Economics and Technology, University of Peloponnese, 22100 Tripoli, Greece; acb@uop.gr

2 Department of Electrical and Computer Engineering, Isfahan University of Technology, Isfahan 84156, Iran; gholami@iut.ac.ir

3 Metro-, Access and Inhouse Systems Group, Photonic Networks and Systems Department, Fraunhofer Heinrich Hertz Institute, Einsteinufer 37, 10587 Berlin, Germany; volker.jungnickel@hhi.fraunhofer.de

4 Department of Mathematics, Physics and Electrical Engineering, Northumbria University, Newcastle upon Tyne NE1 8ST, UK; z.ghassemlooy@northumbria.ac.uk

* Correspondence: kyianno@uop.gr; Tel.: +30-2710-372204

Citation: Boucouvalas, A.C.; Yiannopoulos, K.; Gholami, A.; Jungnickel, V.; Ghassemlooy, Z. Special Issue on Light

Communications: Latest Advances and Prospects. Appl. Sci. 2022, 12, 2349. https://doi.org/10.3390/ app12052349

Received: 25 December 2021

Accepted: 16 February 2022

Published: 24 February 2022

Publisher's Note: MDPI stays neutral with regard to jurisdictional claims in published maps and institutional affiliations.

Copyright: (c) 2022 by the authors Licensee MDPI, Basel, Switzerland. This article is an open access article distributed under the terms and conditions of the Creative Commons Attribution (CC BY) license (https:// creativecommons.org/licenses/by/ $4.0 /)$.

\section{Introduction}

The wireless information carrier shift from the radio frequencies to optical frequencies has been studied in increasing detail since the invention of the laser in the 1960s. With the advent of optical fiber communications and their global use, we have seen a growing interest in the field of optical wireless communications (OWC) for front- and back-haul access networks and beyond as part of fifth-generation (5G) wireless networks. OWC has found niche applications in a wide range of systems, from short-range indoor to long-range outdoor and very long deep space and inter-satellite links [1]. New components, such as visible light-emitting sources, image sensors, advanced signal processing techniques, multi-input multi-output, and machine learning are pushing the OWC technology into new application areas such as vehicular communications, smart environments, medical, manufacturing, etc., as part of 5G wireless networks and the emerging 6G networks and beyond.

This Special Issue was put together with the goal of bringing together the latest research and development in wireless light communications. A total of 53 submissions were received, and 24 were selected for publication in this Special Issue. The published works present novel results mainly on outdoor and indoor applications of light communications, while a few papers also address broader areas. We briefly present these works in the following sections.

\section{Outdoor Systems}

Starting with outdoor systems, the first four papers focused on the impact of atmospheric effects on the performance of free space optics (FSO) links. Androutsos and co-authors [2] have investigated a single-input multiple-output (SIMO) FSO system with wavelength, spatial, or time diversity at the receiver along with the on-off keying (OOK) and L-PPM (pulse position modulation), together with the optimal combining technique. The turbulence effect, modeled using the mixture Gamma distribution and a non-zero boresight pointing error model, are also investigated. The authors have evaluated the performance of the system by deriving novel mathematical expressions for the outage probability and the average bit error rate performance. Li et al. have considered coherent FSO systems [3] and propose a new method for the measurement and estimation of the phase offset introduced by the atmospheric channel. The method combines the existing 
Viterbi-Viterbi phase offset estimation method with Kalman filter, which leads to lowering the estimation error by no less than $60 \%$, even when the signal to noise ratio (SNR) is low.

Boucouvalas, et al. [4] have discussed the performance of optically amplified FSO systems when an orthogonal modulation format such as PPM is utilized to partially mitigate atmospheric-induced fading. Novel analytical approximations are presented for the average bit error rate in these systems, which are shown to be in good agreement with the exact values. This theoretical work also addresses the applicability of diversity reception, identifying fading scenarios where multiple amplifiers can be used despite the associated increase in optical noise. Khandakar and co-authors [5] have conducted experimental research on the effects of weather conditions and the chemical composition of the atmosphere on FSO communications. The authors set up their experiment in Qatar, a region with arid and harsh weather, and monitored the system performance during the four seasons. Their results indicate that the FSO link performs better in winter than in summer, mainly since higher temperatures during summer introduce increased atmospheric scintillations.

The next two papers also report on the impact of atmospheric effects on FSO communications, with the addition of intermediate relays between the transmitter and the receiver. Taher, et al. [6] have studied the performance of three relay selection schemes (source to relay, relay to destination, and source to destination). The obtained results show that no optimum technique exists for all channel conditions, and the relay selection algorithm needs to take into account the severity of atmospheric induced fading and the pointing errors. Elbawab, et al. [7] have evaluated the performance of a relay-assisted FSO communication system with a blind detection scheme. The authors derive novel analytical expressions for the average bit error rate and demonstrate that this technique performs similar to more complex systems requiring channel state information. The optimal relay placement is also investigated using the analytical expressions.

A promising future practical application of outdoor FSOs, namely, space optical communications, is the topic of the next two papers. Li and co-researchers [8] have analyzed the technique of aperture averaging with a goal to partially correct the adverse effects of atmospheric turbulence in a coherent LEO satellite-to-ground binary phase shift keying system. The authors collected data on the channel gain with the increasing aperture sizes, confirming that the performance is initially improved. However, wave front aberrations appear in very large apertures, and an adaptive optics system needs to be installed so as to compensate for the wave-front aberrations. Huang, et al. [9] have discussed the detection of high-orbit targets, which has applications in the establishment of FSO links. They have developed a detection model based on the SNR of the target and verify it via the assembly of an infrared-K band photoelectric system. The detection range is in the order of $10^{4} \mathrm{~km}$, and the results indicate that the short-wave infrared bands have better imaging effects.

The last two papers of this section also discuss important aspects of outdoor systems, albeit non-communication ones. Liu, et al. [10] have developed a novel mode-field matching model, motivated by the problem of efficiently coupling light from FSO systems to optical fibers. Their mathematical model is based on Zernike polynomials, and its predictions are validated through experimental results. Finally, Deng and co-researchers [11] have presented the improvements on a tracking control system, which is based on a microelectro-mechanical systems (MEMS) accelerometer. The authors proposed a modified disturbance observer structure to alleviate the limitations of the aforementioned tracking system at low frequencies and implement the corresponding controller to experimentally validate its performance.

\section{Indoor Systems}

In this area, 10 excellent papers were accepted, of which 9 are on the different aspects of visible light communications (VLC). The first paper by Witas, et al. [12] presents a mobile receiver that uses several photodetectors to provide omnidirectional receiving capability. The photodetectors are fixed to truncated pyramid walls. The receiver was evaluated experimentally for indoor application using bit error rate measurements and comparing 
signal-to-noise ratio parameters for various photodetector numbers. The next two papers discuss the importance of using synchronization for the orthogonal frequency division multiplexing (OFDM) scheme and a pre-equalizer in VLC systems. Offiong, et al. [13] have used a single pilot sequence to achieve both frame synchronization and the peak-to-average power ratio (PAPR) reduction in the optical OFDM scheme. The two direct-current biased optical (DCO)-OFDM and asymmetrically clipped (ACO)-OFDM are discussed in this research. They show that this scheme gives precise frame synchronization at the SNR as low as $4 \mathrm{~dB}$. Khadr and co-authors [14] have aimed to extend the limited bandwidth of phosphorescent white LEDs for a multiple-input single-output (MISO) VLC system introducing a pre-equalizer circuit, with results demonstrating a $28 \%$ bandwidth enhancement. Moreover, they proposed a model for LED arrangement to improve illumination uniformity along the room.

The next paper discusses the interference suppression in a MIMO VLC system. Forkel, et al. [15] have proposed the use of a liquid-crystal display (LCD) as a receiver-side filter unit to focus the field-of-view of the receiver on a specific light source and thereby suppress interference. Interference due to the ambient light, modulated interference and multiaperture interference are introduced and signal-to-interference ratio improvements are derived using experimental results. For MIMO communications, the potential of the proposed interference reduction method is also demonstrated.

The following two papers have focused on the visible light-based positioning for indoor applications. The first paper by Firoozabadi, et al. [16] proposes a new frequency modulated VLC system for localization in underground mining, which is based on a threedimensional trilateration localization scheme. The obtained results show the superiority of the proposed method in comparison with the traditional short range radio frequency technologies. Tran, et al. [17] proposed a VLC-based positioning method using dualfunction machine learning algorithms. Their predicted results show that the proposed solution increases positioning accuracy under the negative effect of multipath reflections and decreases the computational time.

The last 4 papers in this section have focused mostly on the resource allocation and energy efficiency in VLC. Sadat, et al. [18] have investigated the power domain nonorthogonal multiple access (PD-NOMA) method to enhance the spectral efficiency of VLC systems downlink. The bit error rate (BER) and the achievable rate performance of the non-cooperative and cooperative PD-NOMA under a perfect channel state information are investigated and moreover, analytical BER estimation is verified by Monte Carlo simulation. Bai and co-authors [19] have proposed a low-complexity multi-cell resource allocation algorithm with a near-optimal system throughput to resolve the conflict between the high system throughput and low complexity of indoor VLC ultra-dense networks. By the simulation results, authors show that the proposed method achieves an improvement of $57 \%$ in performance in terms of the average system throughput and improvement of $67 \%$ in performance in terms of the quality of service (QoS) guarantee against the required data rate proportion allocation method.

Msongaleli, et al. [20] have proposed a non-contention bandwidth assignment protocol called the adaptive polling medium access control (APMAC) protocol for optical wireless networks. The APMAC protocol involves association, data transmission, and dissociation phases. While assigning bandwidth to the visible light nodes (VLNs), the visible light access point establishes a polling table that contains the identity, buffer size, and round-trip time of each VLN that issued a bandwidth request. The protocol is investigated numerically in terms of average waiting time, packet collision, system utilization, and fairness. Finally, Wang, et al. [21] have proposed a channel and bit adaptive power control strategy for uplink non-orthogonal multiple access VLC systems by jointly considering the channel state information and the transmission bit rate. Under this adaptive power control strategy, it is proved that the received signal at the photodiode receiver constitutes a sizable pulse amplitude modulation constellation and low-complexity maximum likelihood detection is admitted. 


\section{Components and Networks}

Four papers on reflecting on the components and networks of optical communications were accepted. The first paper by Boucouvalas, et al. [22] is a review of the resonant transmission line method, which enables the design of elliptical and eccentric core fibers as well as "holey" photonic crystal fibers. Wang, et al. [23] have presented a broadband planar lightwave circuit splitter with a variable splitting ratio that is designed for signal distribution in passive optical network. Li, et al. [24] have reported pulse-shaping-based optical transmission for a 128-QAM (quadrature amplitude modulation) DWDM (dense wavelength division multiplexing) system that has a potential capacity equal to 904 Gbps per channel. Finally, Abd El-Mottaleb, et al. [25] have presented the analysis and evaluation of a spectral amplitude coding optical code-division multiple-access system with the enhanced double-weight codes as signature codes.

\section{Closing Remarks}

The ongoing interest in light communications has been the driving force behind this Special Issue. The potential of this technology and its possible applications that range from personal to space communication systems will hopefully keep us occupied in the years to come. We sincerely hope that you will enjoy reading this Special Issue as much as we enjoyed compiling it!

Author Contributions: Writing-original draft preparation, A.C.B., K.Y., A.G., V.J. and Z.G.; writingreview and editing, A.C.B., K.Y., A.G., V.J. and Z.G. All authors have read and agreed to the published version of the manuscript.

Funding: This research received no external funding.

Institutional Review Board Statement: Not applicable.

Informed Consent Statement: Not applicable.

Data Availability Statement: The study did not report any data.

Acknowledgments: We would like to thank the authors for their submissions to this Special Issue and the effort they put into their work. This publication would not have been possible without the contribution of peer-reviewers; their comments were invaluable for the improvement of the presented papers. The MDPI staff are to be commended: They extended the invitation for this Special Issue and monitored every step of the processes towards its successful completion.

Conflicts of Interest: The authors declare no conflict of interest.

\section{References}

1. Ghassemlooy, Z.; Arnon, S.; Uysal, M.; Xu, Z.; Cheng, J. Emerging Optical Wireless Communications-Advances and Challenges. IEEE J. Select. Areas Commun. 2015, 33, 1738. [CrossRef]

2. Androutsos, N.A.; Nistazakis, H.E.; Stassinakis, A.N.; Sandalidis, H.G.; Tombras, G.S. Performance of SIMO FSO Links over Mixture Composite Irradiance Channels. Appl. Sci. 2019, 9, 2072. [CrossRef]

3. Li, H.; Huang, Y.; Wang, Q.; He, D.; Peng, Z.; Li, Q. Phase Offset Tracking for Free Space Digital Coherent Optical Communication System. Appl. Sci. 2019, 9, 836. [CrossRef]

4. Boucouvalas, A.C.; Sagias, N.C.; Yiannopoulos, K. Accurate Evaluation of the Average Probability of Error of Pulse Position Modulation in Amplified Optical Wireless Communications under Turbulence. Appl. Sci. 2019, 9, 749. [CrossRef]

5. Khandakar, A.; Touati, A.; Touati, F.; Abdaoui, A.; Bouallegue, A. Experimental Setup to Validate the Effects of Major Environmental Parameters on the Performance of FSO Communication Link in Qatar. Appl. Sci. 2018, 8, 2599. [CrossRef]

6. Taher, M.A.; Abaza, M.; Fedawy, M.; Aly, M.H. Relay Selection Schemes for FSO Communications over Turbulent Channels. Appl. Sci. 2019, 9, 1281. [CrossRef]

7. Elbawab, M.; Abaza, M.; Aly, M.H. Blind Detection for Serial Relays in Free Space Optical Communication Systems. Appl. Sci. 2018, 8, 2074. [CrossRef]

8. Li, H.; Huang, Y.; Wang, Q.; He, D.; Peng, Z.; Li, Q. Performance Analysis of Satellite-to-Ground Coherent Optical Communication System with Aperture Averaging. Appl. Sci. 2018, 8, 2496. [CrossRef]

9. Huang, Z.; Huang, R.; Xue, X. Analysis of SNR for High-Orbit Target Detected by Ground-Based Photoelectric System. Appl. Sci. 2018, 8, 2604. [CrossRef] 
10. Liu, Y.; Wang, J.; Ma, L.; Gao, S.; Guo, C.; Yao, K.; Sheng, L. The Effect of Spatial Mode Distribution on Coupling Efficiency of Single-Mode Fiber: Theoretical Analysis and Experimental Verification. Appl. Sci. 2019, 9, 3296. [CrossRef]

11. Deng, J.; Ren, W.; Zhang, H.; Luo, Y.; Zhou, X.; Mao, Y. A Modified Disturbance Observer Structure Based on Acceleration Measurement for Disturbance Suppression in Tracking Control System. Appl. Sci. 2018, 8, 1571. [CrossRef]

12. Witas, K.; Nedoma, J. Free Space Optic Receiver with Strongly Overlapped Photodetectors' Field of View. Appl. Sci. 2019, 9, 343. [CrossRef]

13. Offiong, F.B.; Sinanović, S.; Popoola, W.O. Pilot-Aided Frame Synchronization in Optical OFDM Systems. Appl. Sci. 2020, 10, 4034. [CrossRef]

14. Khadr, M.H.; Abd El Aziz, A.; Fayed, H.A.; Aly, M. Bandwidth and BER Improvement Employing a Pre-Equalization Circuit with White LED Arrays in a MISO VLC System. Appl. Sci. 2019, 9, 986. [CrossRef]

15. Forkel, G.J.M.; Krohn, A.; Hoeher, P.A. Optical Interference Suppression Based on LCD-Filtering. Appl. Sci. 2019, 9, 3134. [CrossRef]

16. Dehghan Firoozabadi, A.; Azurdia-Meza, C.; Soto, I.; Seguel, F.; Krommenacker, N.; Iturralde, D.; Charpentier, P.; Zabala-Blanco, D. A Novel Frequency Domain Visible Light Communication (VLC) Three-Dimensional Trilateration System for Localization in Underground Mining. Appl. Sci. 2019, 9, 1488. [CrossRef]

17. Tran, H.Q.; Ha, C. Improved Visible Light-Based Indoor Positioning System Using Machine Learning Classification and Regression. Appl. Sci. 2019, 9, 1048. [CrossRef]

18. Sadat, H.; Abaza, M.; Gasser, S.M.; ElBadawy, H. Performance Analysis of Cooperative Non-Orthogonal Multiple Access in Visible Light Communication. Appl. Sci. 2019, 9, 4004. [CrossRef]

19. Bai, X.; Li, Q.; Tang, Y. A Low-Complexity Resource Allocation Algorithm for Indoor Visible Light Communication Ultra-Dense Networks. Appl. Sci. 2019, 9, 1391. [CrossRef]

20. Msongaleli, D.L.; Kucuk, K.; Kavak, A. Adaptive Polling Medium Access Control Protocol for Optic Wireless Networks. Appl. Sci. 2019, 9, 1071. [CrossRef]

21. Wang, Z.-Y.; Yu, H.-Y.; Wang, D.-M. Channel and Bit Adaptive Power Control Strategy for Uplink NOMA VLC Systems. Appl. Sci. 2019, 9, 220. [CrossRef]

22. Boucouvalas, A.C.; Papageorgiou, C.D.; Georgantzos, E.; Raptis, T.E. Resonant Transmission Line Method for Unconventional Fibers. Appl. Sci. 2019, 9, 270. [CrossRef]

23. Wang, H.-T.; Chen, C.-F.; Chi, S. A Numerical Solution for Broadband PLC Splitter with Variable Splitting Ratio Based on Asymmetric Three Waveguide Structures. Appl. Sci. 2019, 9, 1892. [CrossRef]

24. Li, G.; Li, J. A Pulse Shaping Based Optical Transmission System of 128QAM for DWDM with N × 904 Gbps. Appl. Sci. 2019, 9, 988. [CrossRef]

25. Abd El-Mottaleb, S.A.; Fayed, H.A.; Abd El-Aziz, A.; Metawee, M.A.; Aly, M.H. Enhanced Spectral Amplitude Coding OCDMA System Utilizing a Single Photodiode Detection. Appl. Sci. 2018, 8, 1861. [CrossRef] 